\title{
Screening of miRNA target genes in coronary artery disease by variational Bayesian Gaussian mixture model
}

\author{
XIAO-LIN MA ${ }^{1 *}$, XU YANG ${ }^{2 *}$ and RUI FAN ${ }^{3}$ \\ ${ }^{1}$ Department of Cardiology, The People's Hospital of Xuancheng City, Xuancheng, Anhui 242000; \\ ${ }^{2}$ Department of Cardiovascular Disease, The Second People's Hospital of Yunnan Province, Kunming, \\ Yunnan 650021; ${ }^{3}$ Department of Cardiology, Xinjiang Changjizhou People's Hospital, \\ Changjizhou, Xinjiang Uygur Autonomous Region 831100, P.R. China \\ Received May 18, 2018; Accepted January 14, 2019
}

DOI: $10.3892 /$ etm.2019.7195

\begin{abstract}
Coronary artery disease (CAD) is a leading cause of death, and microRNAs (miRNAs) are widely involved in physiological and pathological processes of CAD. We chose the targetscore method calculated via the variational Bayesian Gaussian mixture model (VB-GMM) as the prediction method of target genes. By observing the density overlap, we selected the thresholds of miRNA-1 and miRNA-155. In total, 18 target genes of miRNA-1, and 19 target genes of miRNA-155 were identified. The threshold of miRNA-146a was selected using the $\log \mathrm{FCl}$ value, and 16 target genes were screened out. In this study, our major contribution was to predict the target messenger RNAs (mRNAs) of the chosen miRNAs with the gene expression profiles, which can effectively reduce the workload of screening. Although the validated genes constituted only a small part in the final prediction results, it is a good sign for research in the future. It means that we could provide new research aims for future studies focusing on miRNA regulatory mechanisms.
\end{abstract}

\section{Introduction}

Coronary artery disease (CAD) is a leading cause of death worldwide. Atherosclerosis is an important cause for the development of CAD. In addition, factors including age, sex, hypertension, dyslipidemia, smoking, and diabetes, can increase the risk of CAD (1). CAD increases the occurrence

Correspondence to: Dr Rui Fan, Department of Cardiology, Xinjiang Changjizhou People's Hospital, 303 Yan'an North Road, Changjizhou, Xinjiang Uygur Autonomous Region 831100, P.R. China

E-mail: changjifanrui@163.com

${ }^{*}$ Contributed equally

Key words: human miRNA, target messenger RNA, variational Bayesian Gaussian mixture model, coronary artery disease of myocardial infarction, which may lead to serious complications or even death if the patient has not been treated timely and effectively. Except for the development of efficient drugs, the progression of early prediction and diagnosis are also the direction for CAD prevention. Many studies indicate that microRNAs (miRNAs) are widely involved in physiological and pathological processes of CAD (2-8).

miRNAs are a class of small non-coding RNAs first identified in 1993. miRNAs could either initiate translational inhibition or degradation of messenger RNAs (mRNAs), and adjust gene expression at the post-translational stage, by binding to the 3 '-untranslated region of target mRNAs. Compared with protein-based biomarkers, miRNAs have some good characteristics, including stability in the circulation, higher sensitivity, higher specificity, tissue- and pathology-specific regulation, to be the circulating biomarkers. Previosu findings showed that numerous miRNAs, such as miRNA-1 (2), miRNA-19 (3), miRNA-92 (4), miRNA-133 (5), miRNA-146a (6), miRNA-155 (7) and miRNA-199 (8) are associated with CAD. However, each miRNA has numerous target mRNAs, and the effective recognition of the interaction remains a challenge, limiting the application in CAD prediction. Therefore, bioinformatic methods have been developed and used to predict the effective miRNA-mRNA interaction.

Original TargetScan software was developed by Lewis et $a l$, and it is the first algorithm to predict miRNA targets in vertebrates (9). The first edition of TargetScan obtained miRNA sequences from the Rfam database. The TargetScan method generated a 3'-UTR dataset for mammalian and vertebrate genes to confirm miRNA-target interactions. The TargetScan method examined the data of seed matches including the expected frequency, the observed count and the predicted free energy of the seed to assign a $\mathrm{Z}$ score to each 3'-UTR. However, the seed matches were not always sufficient to confer repression. The grade of repression was extremely unstable in different UTR contexts if repression occurred. In order to avoid this problem, Friedman et al created a modified algorithm known as TargetScan probabilities of conserved targeting (PCT) for quantitatively valuing site preservation (10). The new method incorporates the phylogenetic tree based on the UTR genomic regions, and assesses motif conservative by branch-length metric. Additionally, TargetScan 
PCT widely improved the computation of the conservation-specific backgrounds of single seed-match sites. In 2014, Nam et al developed an advanced prediction model based on TargetScan, termed weighted context+ or wContext+ (11). We termed this new TargetScan method, as targetscore. This algorithm primarily differed from the other TargetScan algorithm in three aspects. Firstly, the model of targetscore was especially designed for miRNA-overexpression experiments to explore targets of a specific miRNA in a particular cell condition. Secondly, the model inferred miRNA-targets solely based on their individual high-dimensional modes of expression fold-changes and sequence features. Finally, this method operated on the whole gene expression profile to more closely imitate the overall likelihood rather than the previous TargetScan method. In summary, the targetscore algorithm could afford independent and supplementary information by PCT values and the context scores which are useful for predicting the target gene of each miRNA.

Thus, the targetscore algorithm was used to predict the target gene for miRNA-1, miRNA-155 and miRNA-146a, which are closely related to the pathogenesis of CAD.

\section{Materials and methods}

Gene expression profile microarray data. The gene expression profile dataset no. GSE7638, stored in the Gene Expression Omnibus (GEO) database (http://www.ncbi.nlm.nih.gov/geo/) by Pascal and Meier based on the GPL571 platform, was analyzed by bioinformatics analysis in our study. The dataset is comprised of 160 samples, including 110 cases of total RNAs obtained from patients with CAD and 50 cases of total RNAs obtained from healthy individuals. The ratio of male to female is moderate in this dataset. We used the pre-processing software to integrate data after the different samples were grouped.

Gene expression profile matrix. The expression values of the gene expression profile were pre-processed to facilitate the calculation of the differential expression of genes. The values after pre-processing exclude the missing, the unvaried, the maximum and the minimum values. We removed the unsuitable values to obtain the relative expression and form a new expression dataset. The database of gene pre-treatment was selected as hgu133a. The first step in data processing was to merge multiple probes of the same gene, and the average of multiple probes was chosen as the expression of the gene. The second step was to modify the annotation information (rename the columns to CAD and control group). Finally, we renamed the row of the expression matrix to GENESYMBOL after reprocessing the gene expression values.

After the gene expression was pre-processed, we used the limma package to calculate the $\log \mathrm{FC}$ values for all genes. The limma package has been certified a common choice for the analysis of data from the experiments on microarray. The functions of the package have expanded significantly in the differential expression of RNA sequencing and the analysis of expression profiles. According the gene expression differences of CAD and control group, the $\log \mathrm{FC}$ calculated by limma package was as a priori value for the experiment (Fig. 1). From TargetScan website we entered each miRNA to obtain the TargetScan context score (TSCS)

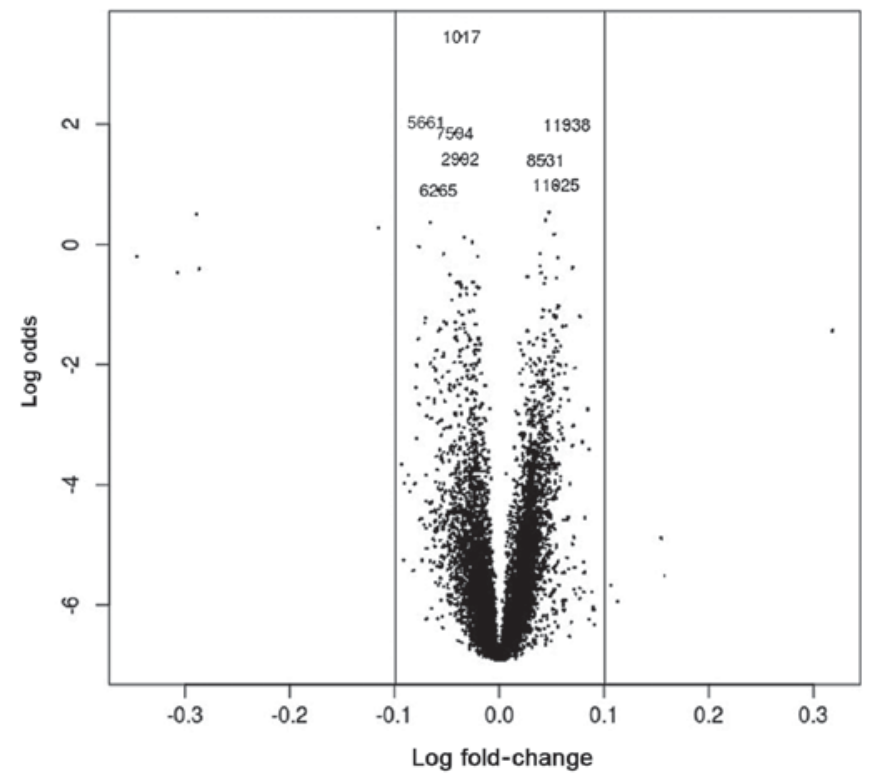

Figure 1 . The $\log \mathrm{FC}$ values of all the genes were calculated by the limma package. Each black dot in the graph represents the $\log F C$ value of a gene.

and PCT values of each gene. Combining the data search in the website with the $\log \mathrm{FC}$ obtained in the gene expression profiling experiment, we used the variational Bayesian Gaussian mixture model (VB-GMM) to calculate the targetscore of the genes. The miRNA target genes were predicted by the targetscore value.

Calculation of the targetscore. The VB-GMM model is rearranged according to the Bayesian GMM model optimized by Li et al (12). The target mRNAs were predicted based on the sequence scores using the integrated miRNA overexpression data and other predictive methods. The miRNA-mRNA interaction is estimated by the posterior probability distribution of the observed variables, and the targetscore value is calculated as a weighted sigmoid-transformed fold-change. Variational Bayesian EM means the variational Bayesian expectation maximization. It is based on variational inference to find the edge distribution of minimized Kullback-Leibler distance to approximate the joint distribution, and use the mean field to reduce the complexity of the joint estimation. Variational Bayesian is a technique for approximating computational complexity in Bayesian estimation and machine learning. It also used the maximum a posteriori (MAP), that is, with the single most probable parameter value instead of a complete Bayesian estimate. This is why we consider it as an extension of the EM algorithm to some extent. In addition, the variational Bayesian also obtains the optimal solution through a series of mutually dependent equations. We obtained the TSCS, PCT and $\log \mathrm{FC}$ values of the genes in the experimental data, and deduced the maximum likelihood (ML) value by VB-GMM as the posterior probability of each gene. The targetscore values were calculated by the following formula to quantify the target genes of miRNA.

$$
\text { targetscore }=\frac{1}{1+\mathrm{e}^{x}} \times \bar{p}
$$

In the formula, $\mathrm{x}$ refers to the $\log \mathrm{FC}$ value of the gene, and $\mathrm{p}$ refers to the posterior value inferred. 
A

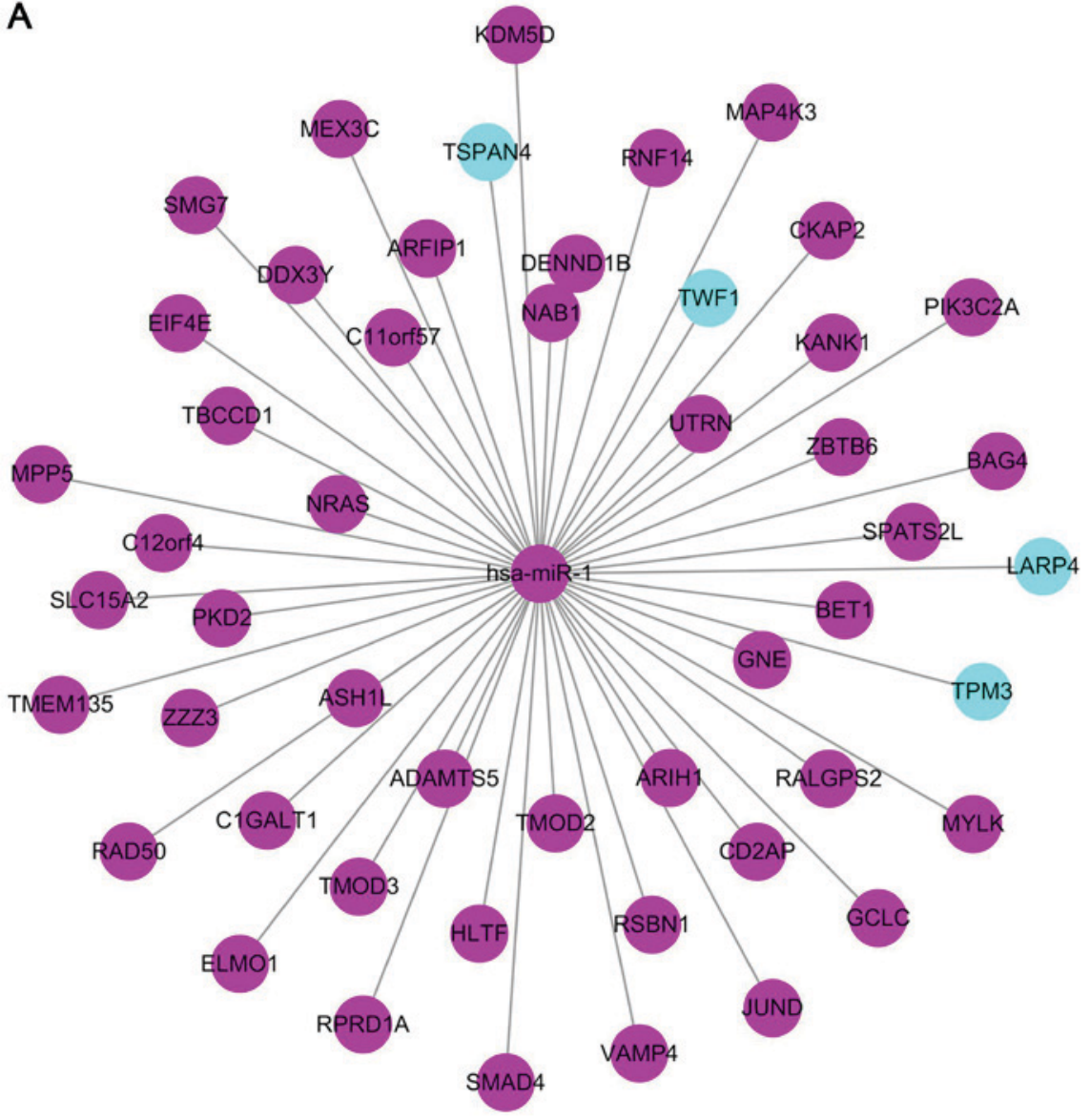

B

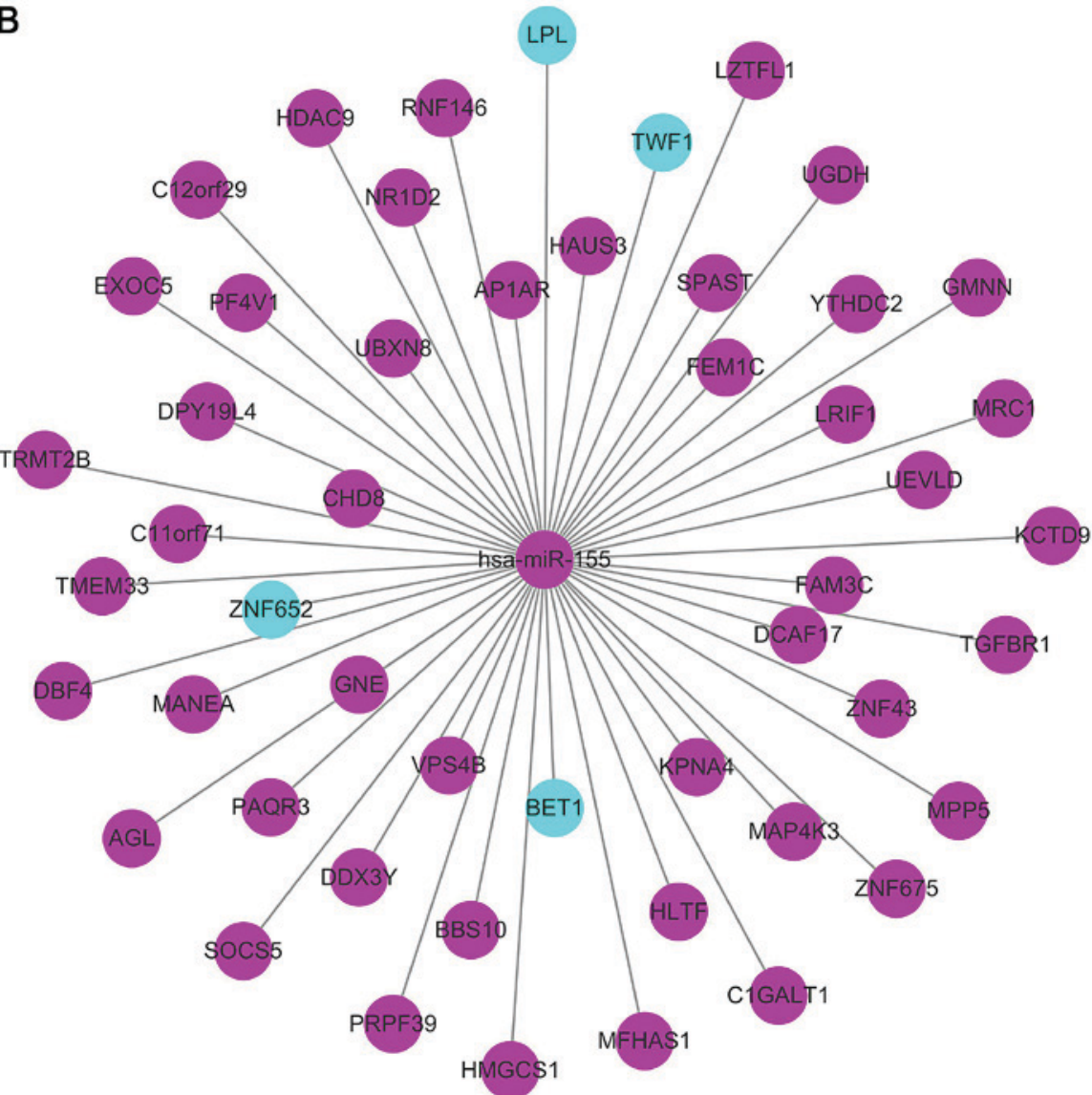

Figure 2 (A and B). The three charts show the top 50 target genes of miRNA-1, miRNA-155 and miRNA-146a. The primary screening target genes of (A) miR-1, (B) miR-155. The values between the miRNAs and the predicted genes are the targetscore and the blue-labeled genes are the validated target genes. miRNA, microRNA. 


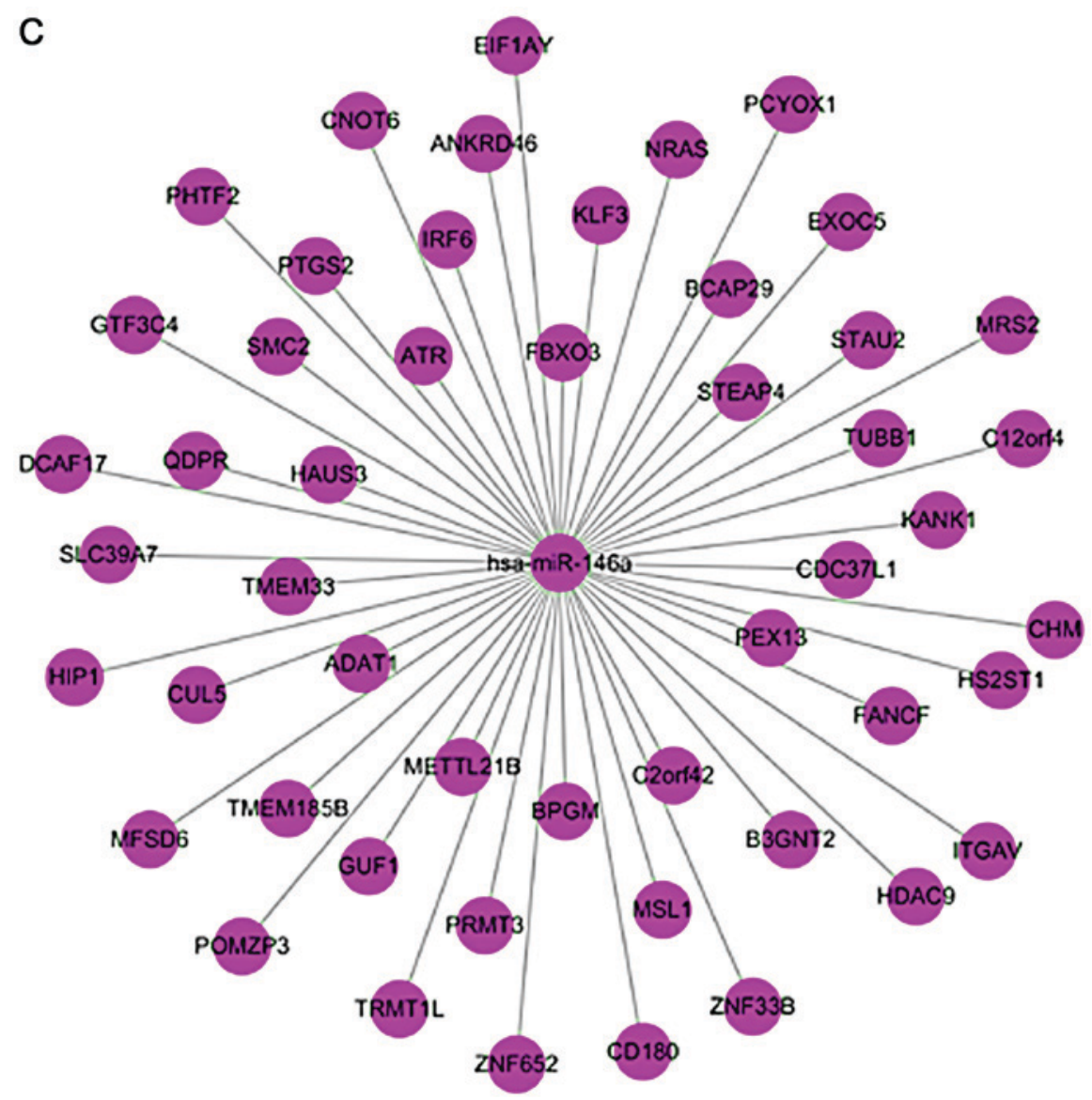

Figure 2 C. Continued. These three charts show the top 50 target genes of miRNA-1, miRNA-155 and miRNA-146a. (C) The primary screening target genes of miR-146a. The values between the miRNAs and the predicted genes are the targetscore and the blue-labeled genes are the validated target genes. miRNA, microRNA.

\section{Results}

Preliminary screening with the targetscore value. In the results, we predicted 362 miRNA-1-related gene targetscore values $>0.333$, of which 47 genes were validated. After the prediction of miRNA-155, there were 648 gene targetscore values $>0.333$, of which 63 genes were validated. The miRNA-146a had 428 target genes of the targetscore value $>0.333$ and only 1 gene was validated. According to the targetscore value from high to low order, we extracted the top 50 genes. Of the first 50 genes of miRNA-1, the validated genes were $L A R P 4, T S P A N 4, T W F 1$ and TPM3. In the first 50 genes of miRNA-155, the validated genes are $L P L, Z N F 652, B E T 1$ and $T W F 1$. There were only non-validated genes in the first 50 genes of miRNA146a (Fig. 2).

Selection of the targetscore threshold and target genes. To further increase the credibility of the target genes and make accurate screening results, we analyzed the density overlap between the targetscore distribution of the validated and the non-validated target genes to make the screening result more exact. We counted the number of the validated and the non-validated target genes in each targetscore value and drew a curve graph for convenient observation (Fig. 3). In the end, we chose the little overlap between the two distributions as the targetscore threshold.
We observed the overlap on the miRNA-1 and found that the distribution overlap decreases when targetscore $=0.341$. Therefore, we selected targetscore $=0.341$ as the threshold for screening the target genes for miRNA-1. We observed the miRNA-155 target gene overlap of targetscore distribution in the same way. When targetscore is 0.344 , the overlap was significantly reduced, so we chose it as the standard for filtering inaccurate miRNA-155 target genes. We screened miRNA-1 and miRNA-155 target genes again with the thresholds selected by analyzing the density distribution of the genes. There were a total of 18 miRNA-1 target genes which are $D D X 3 Y, K D M 5 D, T M O D 3, Z B T B 6, M A P 4 K 3, L A R P 4$, RPRD1A, TSPAN4, UTRN, CD2AP, CAGALT1, C12orf4, BET1, HLTF, SMAD4, MPP5, KANK1, and CKAP2 (Table I). miRNA-155 has 19 target genes, including PF4V1, LPL, DDX3Y, LRIF1, LZTFL1, KCTD9, PRPF39, RNF146, FEM1C, MAP4K3, PAQR3, C11orf71, FAM3C, BBS10, HMGCS1, TRMT2B, YTHDC2, UGDH, and ZNF652 (Table II).

Unlike miRNA-1 and miRNA-155, there is only one validated gene in the miRNA-146a prediction result. So the re-screening method chosen to predict miRNA-146a is different from the other two miRNAs. We decided to re-screen the target genes of miRNA-146a according to the value of the $\mid \operatorname{logFCl}$. Based on past experience, we regarded $\mid \log \mathrm{FCl} \geq 0.05$ as the standard for re-screening target genes of miRNA-146a, and the targetscore threshold is 0.343 . The genes, including 

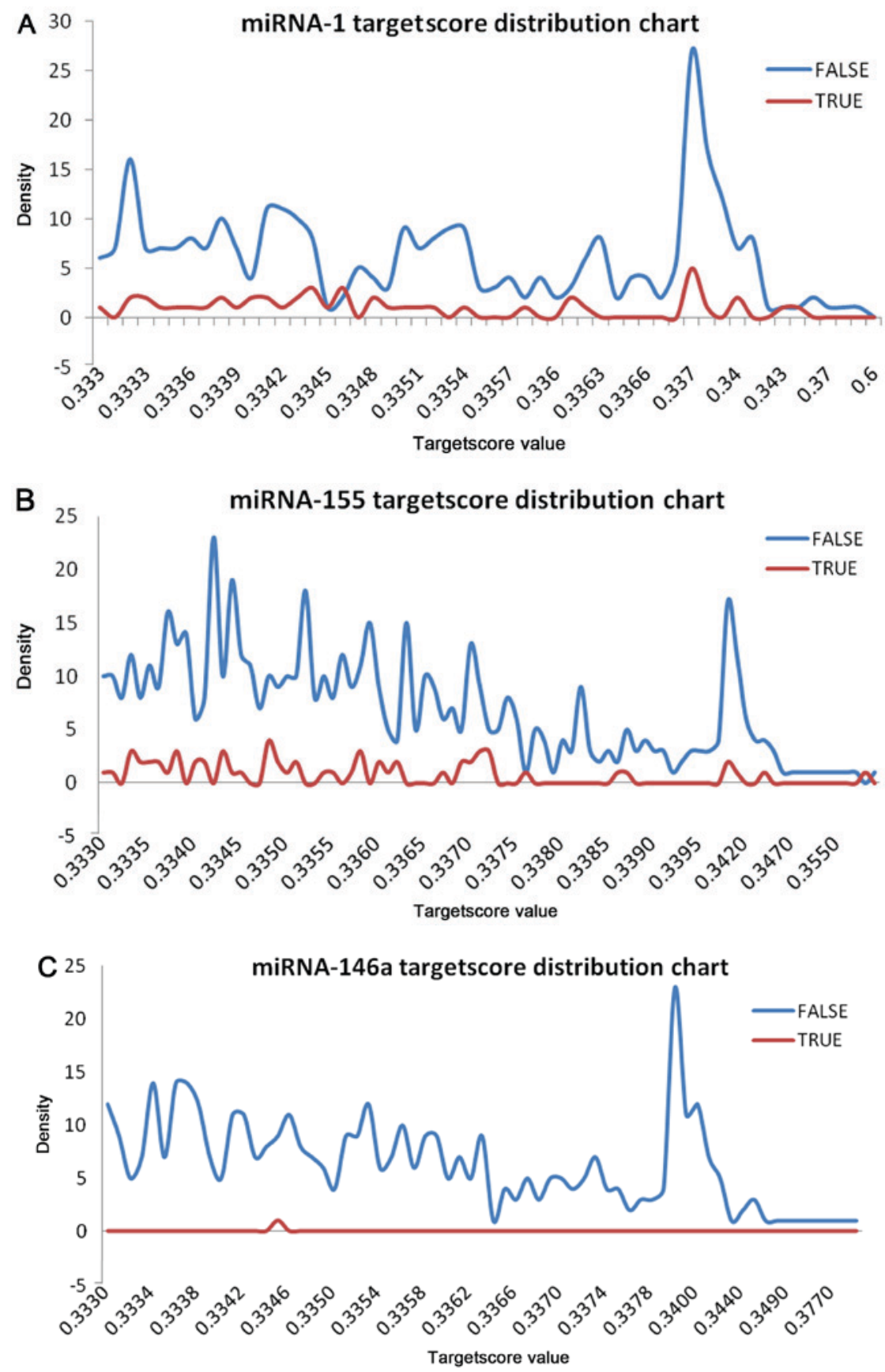

Figure 3. The density distribution of validated and non-validated of the relevant gene targetscore is a criterion for filtering the lack of confidence in the target gene. The distribution of (A) miRNA-1, (B) miRNA-155, and (C) miRNA-146a targetscore. False refers to non-validated genes (in the past there was no exact genetic study reference). True refers to the validation gene (in the past there was a definite genetic study reference). Density of targetscore refers to the number of genes in a targetscore value. miRNA, microRNA.

PTGS2, EIF1AY, GUF1, TUBB1, PCYOX1, CHM, TRMT1L, ANKRD46, STEAP4, B3GNT2, TMEM185B, QDPR, CDC37L1, PRMT3, ZNF652, and DCAF17, were selected as target genes for miRNA-146a (Table III).

Relevant references of validated genes. Some of the predicted genes in the target gene were validated, but most of the genes were not validated. We compiled the relevant literature information of the validated genes into a Table. Since the first 50 target genes of miRNA-146a are non-validated, we listed the literature information only of the validated gene in all the genes (Table IV).

\section{Discussion}

miRNAs have important effect because they could regulate gene expression at the post-transcriptional level by binding to the 3'-UTR of mRNAs. Several scientific gains have found that miRNAs have the function of regulating specific genes. With the increase of the research of CAD diagnosis and prediction by miRNA, we found some meaningful results. Wang et al found that the genetic variant of one of the miRNA-1 target genes binding site was associated with the major adverse cardiovascular events of CAD (2). In the study, researchers compared the target gene COG6 rs9548934 $\mathrm{C} \rightarrow \mathrm{T}$ variant with 
Table I. The miRNA-1 target genes.

\begin{tabular}{|c|c|c|c|c|}
\hline $\begin{array}{l}\text { External } \\
\text { gene ID }\end{array}$ & $\operatorname{LogFC}$ & TSCS & $\begin{array}{l}\text { TargetScan } \\
\text { PCT }\end{array}$ & targetscore \\
\hline$D D X 3 Y$ & -0.28881 & -0.045 & -0.31 & 0.565146 \\
\hline$K D M 5 D$ & -0.28676 & -0.126 & 0 & 0.384165 \\
\hline TMOD3 & -0.07866 & -0.046 & -0.06 & 0.370506 \\
\hline ZBTB6 & -0.06244 & -0.15 & -0.63 & 0.347949 \\
\hline$M A P 4 K 3$ & -0.06196 & -0.298 & -0.56 & 0.347653 \\
\hline LARP4 & -0.05549 & -0.11 & -0.8 & 0.344617 \\
\hline$R P R D I A$ & -0.05434 & -0.065 & -0.05 & 0.344199 \\
\hline TSPAN4 & -0.05361 & -0.248 & -0.62 & 0.343959 \\
\hline UTRN & -0.05173 & -0.207 & -0.6 & 0.343371 \\
\hline$C D 2 A P$ & -0.04958 & -0.332 & -0.89 & 0.342759 \\
\hline CIGALT1 & -0.04657 & -0.219 & -0.4 & 0.341989 \\
\hline C12orf4 & -0.04641 & -0.142 & -0.62 & 0.34195 \\
\hline BETl & -0.04592 & -0.223 & -0.77 & 0.341833 \\
\hline$H L T F$ & -0.04491 & -0.088 & -0.36 & 0.341596 \\
\hline SMAD4 & -0.04413 & -0.088 & -0.7 & 0.34142 \\
\hline MPP5 & -0.04341 & -0.273 & -0.7 & 0.341258 \\
\hline KANKI & -0.04295 & -0.077 & -0.18 & 0.341156 \\
\hline$C K A P 2$ & -0.04248 & -0.103 & -0.12 & 0.341054 \\
\hline
\end{tabular}

miRNA, microRNA; TSCS, TargetScan context score; PCT, probabilities of conserved targeting.

Table II. The miRNA-155 target genes.

\begin{tabular}{|c|c|c|c|c|}
\hline $\begin{array}{l}\text { External } \\
\text { gene ID }\end{array}$ & $\operatorname{LogFC}$ & TSCS & $\begin{array}{c}\text { TargetScan } \\
\text { PCT }\end{array}$ & targetscore \\
\hline PF4VI & 0.113045 & -0.195 & -0.06 & 0.465517 \\
\hline$L P L$ & -0.08697 & -0.04 & -0.07 & 0.416066 \\
\hline$D D X 3 Y$ & -0.28881 & -0.215 & -0.01 & 0.412147 \\
\hline LRIF 1 & -0.07936 & -0.329 & -0.07 & 0.377031 \\
\hline LZTFLI & -0.07061 & -0.192 & -0.07 & 0.355845 \\
\hline KCTD9 & -0.06963 & -0.111 & -0.14 & 0.354505 \\
\hline PRPF39 & -0.06604 & -0.098 & -0.08 & 0.350639 \\
\hline RNF146 & -0.06437 & -0.055 & -0.07 & 0.349273 \\
\hline FEMIC & -0.06326 & -0.208 & -0.06 & 0.348484 \\
\hline$M A P 4 K 3$ & -0.06196 & -0.184 & -0.06 & 0.347653 \\
\hline$P A Q R 3$ & -0.06076 & -0.056 & -0.06 & 0.346964 \\
\hline C11orf71 & -0.05819 & -0.147 & -0.07 & 0.345704 \\
\hline FAM $3 C$ & -0.05812 & -0.074 & -0.07 & 0.345674 \\
\hline BBS10 & -0.0573 & -0.099 & -0.07 & 0.345325 \\
\hline$H M G C S 1$ & -0.05637 & -0.265 & -0.07 & 0.344948 \\
\hline$T R M T 2 B$ & -0.05617 & -0.064 & -0.06 & 0.344872 \\
\hline$Y T H D C 2$ & -0.05569 & -0.141 & -0.06 & 0.34469 \\
\hline$U G D H$ & -0.05498 & -0.089 & -0.07 & 0.34443 \\
\hline ZNF652 & -0.05379 & -0.187 & -0.66 & 0.344021 \\
\hline
\end{tabular}

miRNA, microRNA; TSCS, TargetScan context score; PCT, probabilities of conserved targeting.
Table III. The miRNA-146a target genes.

\begin{tabular}{lllcl}
\hline $\begin{array}{l}\text { External } \\
\text { gene ID }\end{array}$ & LogFC & TSCS & $\begin{array}{c}\text { TargetScan } \\
\text { PCT }\end{array}$ & targetscore \\
\hline PTGS2 & -0.09076 & -0.163 & -0.14 & 0.443186 \\
EIF1AY & -0.307 & -0.2 & 0 & 0.384102 \\
GUF1 & -0.07941 & -0.114 & -0.16 & 0.37725 \\
TUBB1 & 0.089999 & -0.066 & -0.15 & 0.369688 \\
PCYOX1 & -0.06924 & -0.103 & -0.16 & 0.354002 \\
CHM & -0.06777 & -0.049 & -0.16 & 0.352296 \\
TRMT1L & -0.06562 & -0.267 & -0.15 & 0.350279 \\
ANKRD46 & -0.06506 & -0.129 & -0.14 & 0.349811 \\
STEAP4 & -0.06161 & -0.095 & -0.16 & 0.347444 \\
B3GNT2 & -0.06078 & -0.139 & -0.16 & 0.346977 \\
TMEM185B & -0.0586 & -0.204 & -0.16 & 0.345892 \\
QDPR & -0.05846 & -0.115 & -0.14 & 0.345829 \\
CDC37L1 & -0.05836 & -0.148 & -0.13 & 0.345781 \\
PRMT3 & -0.05643 & -0.323 & -0.15 & 0.34497 \\
ZNF652 & -0.05379 & -0.295 & -0.14 & 0.344021 \\
DCAF17 & -0.05333 & -0.108 & -0.16 & 0.343869 \\
\hline
\end{tabular}

miRNA, microRNA; TSCS, TargetScan context score; PCT, probabilities of conserved targeting.

Table IV. The validated target gene references.

\begin{tabular}{lcccc}
\hline miRNA & $\begin{array}{c}\text { Target } \\
\text { gene }\end{array}$ & $\begin{array}{c}\text { Target gene } \\
\text { (Entrez ID) }\end{array}$ & Experiments & $\begin{array}{c}\text { Refs. } \\
\text { (PMID) }\end{array}$ \\
\hline Hsa-miR-1 & LARP4 & 113251 & $\begin{array}{c}\text { Luciferase } \\
\text { reporter assay }\end{array}$ & 20144220 \\
Hsa-miR-1 & TSPAN4 & 7106 & Microarray & 15685193 \\
Hsa-miR-1 & TWF1 & 5756 & Microarray & 15685193 \\
Hsa-miR-1 & TPM3 & 7170 & pSILAC & 18668040 \\
Hsa-miR-155 & LPL & 4023 & pSILAC & 18668040 \\
Hsa-miR-155 & ZNF652 & 22834 & luciferase & 18367535 \\
& & \multicolumn{3}{c}{ reporter assay } \\
Hsa-miR-155 & BET1 & 10282 & pSILAC & 18668040 \\
Hsa-miR-155 & TWF1 & 5756 & pSILAC & 18668040 \\
Hsa-miR-146a & TRAF6 & 7189 & western blotting/ & 18504431 \\
& & \multicolumn{3}{c}{ northern blotting } \\
\hline
\end{tabular}

miRNA, microRNA; pSILAC, pulsed SILAC.

the wide genotype $\mathrm{CC}$, and found that the genetic variant could significantly reduce the risk of CAD. This means that a diminutive variation in the gene miRNA-binding sites may have an influence on numerous target mRNAs, and the polymorphism in miRNA target sites could be modulated in miRNA-1 targeting. The scholars have utilized the bioinformatics tool to confirm that MAP3K10 is the target gene of miRNA-155, and the results proved that targeting MAP3K10 may be a major mechanism for the anti-atherosclerosis effect 
of miR-155 (13). It has been confirmed that miRNAs could control the main cell function to regulate the progression of atherosclerosis. miRNA-146a was reported to be a regulator of the inflammatory process and influence the pathology of CAD (14). From the literature, we found that miRNA-1, miRNA-155 and miRNA-146a were all closely related to the pathological process of CAD. They can control the expression of effective target genes to affect the probability of the major adverse cardiovascular events of CAD. So we selected these three miRNAs for target gene prediction in order to do the preparatory work for clarifying the mechanism of CAD.

In this study, we chose the targetscore method to predict the target genes of miRNAs, and the greatest advantage of this method is that it could be associated with the selected disease to make the results more specific. Screening the accurate target genes not only can make the mechanism of miRNAs in CAD clear, but also can help the future of medicine research and development. After we made the prediction, we found that a large number of the genes in results were validated thus indicating the results were credible. Nevertheless the initial screening of the results were too large, we used the targetscore value for re-screening to make the consequence accurate. First we considered the top 50 genes of the targetscore value as the precise target genes and analyzed them. We found that LARP4, TSPAN4, TWF1 and TPM3 of miRNA-1 were validated. The $L P L, Z N F 652, B E T 1$ and $T W F 1$ of miRNA-155 were also validated. But the first 50 genes of miRNA146a were all non-validated. In order to further refine the results, we used the density overlap between the targetscore distribution of the validated and the non-validated target genes to re-screen. By observing the density overlap, we selected the thresholds of miRNA-1 as targetscore $=0.341$, and the thresholds of miRNA-155 as targetscore $=0.344$. Unlike the other two miRNAs, there was only one validated gene in the miRNA-146a predicted results. Therefore the re-screening method cannot use the density overlap, so we refined its prediction result based on the $\log \mathrm{FCl}$ values (12). In the end, we used the $\log \mathrm{FCl}$ value $>0.05$ as the standard of miRNA-146a exact screening and the targetscore threshold is 0.343 . According to the targetscore threshold, we finally confirmed that the miRNA-1 target genes are DDX3Y, KDM5D, TMOD3, ZBTB6, MAP4K3, LARP4, RPRDIA, TSPAN4, UTRN, CD2AP, CAGALT1, C12orf4, BET1, HLTF, SMAD4, MPP5, KANK1, and $C K A P 2$. The miRNA-155 target genes are PF4V1, LPL, DDX3Y, LRIF1, LZTFL1, KCTD9, PRPF39, RNF146, FEM1C, MAP4K3, PAQR3, C11orf71, FAM3C, BBS10, HMGCS1, TRMT2B, YTHDC2, UGDH, and ZNF652. The miRNA-146a target genes are PTGS2, EIF1AY, GUF1, TUBB1, PCYOX1, CHM, TRMT1L, ANKRD46, STEAP4, B3GNT2, TMEM185B, QDPR, CDC37L1, PRMT3, ZNF652, and DCAF17. In the final result, the LARP4 and TSPAN4 of miRNA-1 as well as the LPL and ZNF652 of miRNA-155 were validated.

We retrieved the references of the validated genes, including LARP4, TSPAN4, LPL and ZNF652, and carefully analyzed them. Takane et al considered that miRNA-1 is an evolutionarily conserved miRNA which controls the expression of a large number of target genes and attempted to explore miRNA-1-mediated gene regulatory mechanisms by analyzing miRNA-target gene pairs (15). They believe that the complementarily between miRNA and 3'-UTR was weak when the method was chosen, so the phylogenetic profiling approach was chosen as the research method. Five miRNA families, including miRNA-1, were extracted from five species of Diptera animals. Firstly, the potential target genes were extracted according to the optimal free-energy information. Then, the sequence of the seed was matched by RNAhybrid software, and the potential binding site of the short RNA in the target sequence was predicted. Finally, the target genes were screened according to the 'enrichment' index. The results show that miRNA-1 downregulates the expression of LARP4. It suggests that miRNAs can regulate their own miRNA pathway by controlling the expression of significant translation factors. Lim et al transfected a double-stranded piece of miRNA-1 into HeLa cells and used microarray to select the mRNAs whose expression profiles were different from the LocusLink database (16). They observed that miRNA-1 is preferentially expressed in the heart and skeletal muscle of mammals, and TSPAN4 is a target gene of miRNA-1. The predictive results confirmed the hypothesis that vertebrate miRNAs have a large number of target genes. Selbach et al indicated that recognizing miRNA-target gene pairs is one of the most critical aspects of understanding life (17). They measured the protein difference between diverse transfected HeLa cells by the pulsed SILAC (pSILAC) method, and finally verified 16 pSILAC genes by western blotting. Yin et al analyzed the gene expression of miRNA-155 by cell experiment, and the target gene was predicted by TargetScan Bayesian method (18). Seven transcriptional regulatory genes including ZNF652 were regulated by miRNA- 155 .

Although the validated genes are only a small part in the final prediction results, our results were obtained by the targetscore method which is more accurate than the TargetScan method. The targetscore method uses the limma package to calculate the $\log \mathrm{FC}$ value as the a priori value. After the gene expression profiling, the $\log \mathrm{FC}$ value was obtained, and it was calculated as the posterior value by VB-GMM model with TSCS and PCT. The targetscore method is more closely related to the disease than the TargetScan method, and the predicted target genes are more accurate. So we believe that genes with high targetscore values are more likely to be the target genes that are regulated by miRNAs during the development and progression of CAD. In this study, our major contribution is to predict the target mRNAs of the chosen miRNAs with the gene expression profiles, which can effectively reduce the workload of screening. Most of the predicted results are non-validated genes, which is a good sign for the research in the future. Because the results predicted by our method could effectively avoid the false-positive, and provide more direction and ideas to research the mechanism of miRNA affecting CAD.

\section{Acknowledgements}

This article was completed in the careful guidance and strong support of Director Jiupei Cheng. The rigorous academic attitude, high professionalism, profound knowledge and innovative spirit of Dean YiWen Wang have had a significant impact on me. I would like to pay special tribute to Dr Zheng Huang for his guidance on the experiments, and also express my heartfelt thanks to Mr. CunMing Fang for his assistance in reference literature search. (X-L Ma). 


\section{Funding}

No funding was received.

\section{Availability of data and materials}

The datasets used and/or analyzed during the current study are available from the corresponding author on reasonable request.

\section{Authors' contributions}

XLM and RF designed the study. XLM was involved in data collection. XY performed the statistical analysis and prepared the figures. XLM and XY drafted the report. RF contributed substantially to its revision. All authors read and approved the final manuscript.

\section{Ethics approval and consent to participate}

Not applicable.

\section{Patient consent for publication}

Not applicable.

\section{Competing interests}

The authors declare that they have no competing interests.

\section{References}

1. Eriksen A, Tillin T, O'Connor L, Brage S, Hughes A, Mayet J, McKeigue P, Whincup P, Chaturvedi N and Forouhi NG: The impact of health behaviours on incident cardiovascular disease in Europeans and South Asians - a prospective analysis in the UK SABRE study. PLoS One 10: e0117364, 2015.

2. Wang L, Zhi H, Li Y, Ma G, Ye X, Yu X, Yang T, Jin H, Lu Z and Wei P: Polymorphism in miRNA-1 target site and circulating miRNA-1 phenotype are associated with the decreased risk and prognosis of coronary artery disease. Int J Clin Exp Pathol 7: 5093-5102, 2014.

3. Lai Y, He S, Ma L, Lin H, Ren B, Ma J, Zhu X and Zhuang S: HOTAIR functions as a competing endogenous RNA to regulate PTEN expression by inhibiting miR-19 in cardiac hypertrophy. Mol Cell Biochem 432: 179-187, 2017.

4. Liu H, Li G, Zhao W and Hu Y: Inhibition of miR-92a may protect endothelial cells after acute myocardial infarction in rats: Role of KLF2/4. Med Sci Monit 22: 2451-2462, 2016.

5. Castoldi G, Di Gioia CR, Bombardi C, Catalucci D, Corradi B, Gualazzi MG, Leopizzi M, Mancini M, Zerbini G, Condorelli G, et al: MiR-133a regulates collagen 1A1: Potential role of miR-133a in myocardial fibrosis in angiotensin II-dependent hypertension. J Cell Physiol 227: 850-856, 2012.
6. Wu ZW, Liu YF, Wang S and Li B: miRNA-146a induces vascular smooth muscle cell apoptosis in a rat model of coronary heart disease via NF-кB pathway. Genet Mol Res 14: 18703-18712, 2015.

7. Kishore R, Verma SK, Mackie AR, Vaughan EE, Abramova TV, Aiko I and Krishnamurthy P: Bone marrow progenitor cell therapy-mediated paracrine regulation of cardiac miRNA-155 modulates fibrotic response in diabetic hearts. PLoS One 8: e60161, 2013.

8. Baumgarten A, Bang C, Tschirner A, Engelmann A, Adams V, von Haehling S, Doehner W, Pregla R, Anker MS, Blecharz K, et al: TWIST1 regulates the activity of ubiquitin proteasome system via the miR-199/214 cluster in human end-stage dilated cardiomyopathy. Int J Cardiol 168: 1447-1452, 2013.

9. Lewis BP, Shih IH, Jones-Rhoades MW, Bartel DP and Burge CB: Prediction of mammalian microRNA targets. Cell 115: 787-798, 2003.

10. Friedman RC, Farh KK, Burge CB and Bartel DP: Most mammalian mRNAs are conserved targets of microRNAs. Genome Res 19: 92-105, 2009.

11. Nam JW, Rissland OS, Koppstein D, Abreu-Goodger C, Jan CH, Agarwal V, Yildirim MA, Rodriguez A and Bartel DP: Global analyses of the effect of different cellular contexts on microRNA targeting. Mol Cell 53: 1031-1043, 2014.

12. Li Y, Goldenberg A, Wong KC and Zhang Z: A probabilistic approach to explore human miRNA targetome by integrating miRNA-overexpression data and sequence information. Bioinformatics 30: 621-628, 2014.

13. Zhu J, Chen T, Yang L, Li Z, Wong MM, Zheng X, Pan X, Zhang L and Yan H: Regulation of microRNA-155 in atherosclerotic inflammatory responses by targeting MAP3K10. PLoS One 7: e46551, 2012

14. Wang Y, Wang X, Li Z, Chen L, Zhou L, Li C and Ouyang DS: Two single nucleotide polymorphisms (rs2431697 and rs2910164) of miR-146a are associated with risk of coronary artery disease. Int J Environ Res Public Health 14: 14, 2017.

15. Takane K, Fujishima K, Watanabe Y, Sato A, Saito N, Tomita M and Kanai A: Computational prediction and experimental validation of evolutionarily conserved microRNA target genes in bilaterian animals. BMC Genomics 11: 101, 2010.

16. Lim LP, Lau NC, Garrett-Engele P, Grimson A, Schelter JM, Castle J, Bartel DP, Linsley PS and Johnson JM: Microarray analysis shows that some microRNAs downregulate large numbers of target mRNAs. Nature 433: 769-773, 2005.

17. Selbach M, Schwanhäusser B, Thierfelder N, Fang Z, Khanin R and Rajewsky N: Widespread changes in protein synthesis induced by microRNAs. Nature 455: 58-63, 2008.

18. Yin Q, McBride J, Fewell C, Lacey M, Wang X, Lin Z, Cameron J and Flemington EK: MicroRNA-155 is an Epstein-Barr virus-induced gene that modulates Epstein-Barr virus-regulated gene expression pathways. J Virol 82: 5295-5306, 2008. International (CC BY-NC-ND 4.0) License. 